COLO-HEP-440

\title{
Determining Lines of Constant Physics in the Confinement Phase of the SU(2) Higgs Model
}

\author{
F \\ Collaboration \\ Francesco Knechtli ${ }^{\dagger}$ \\ Physics Department, University of Colorado, \\ Boulder, CO 80309 USA
}

\begin{abstract}
We present a method for finding lines of constant physics in the confinement phase of the $\mathrm{SU}(2)$ Higgs model on the lattice. The model is considered at finite values of the cut-off where it behaves like an effective field theory with three independent couplings. In particular, a renormalised quantity sensitive to a variation of the bare Higgs quartic self-coupling is constructed from generalised Binder cumulants. Numerical results for the non-perturbative matching of the bare parameters of the model between $\beta=2.2$ and $\beta=2.4$ are presented.
\end{abstract}

COLO-HEP-440

December 1999

$\dagger$ e-mail: knechtli@pizero.colorado.edu 


\section{Introduction}

The conventional bare parameter space of the SU(2) Higgs model [1] is defined by three parameters: $\beta=4 / g^{2}$, where $g$ is the bare gauge coupling, the hopping parameter $\kappa$ and the bare Higgs quartic self-coupling $\lambda$. The continuum limit of the lattice model is at $\beta=\infty$ and $\kappa=1 / 8$. In general, the physics in the continuum limit of a lattice regularised field theory with several relevant couplings depends on the way in which this limit is approached. The approach to the continuum is represented in the bare parameter space by lines on which dimensionless and renormalised ratios $F$ of physical quantities are constant. These lines are called lines of constant physics (LCP) [2].

It is well accepted - supported by the weak gauge coupling expansion and by numerical simulations - that the scalar part of the $\mathrm{SU}(2)$ Higgs model in four spacetime dimensions is a trivial theory [3], which means that in the continuum limit of the $\mathrm{SU}(2)$ Higgs model the renormalised scalar coupling $\lambda_{\mathrm{R}}$ is always zero and therefore there are two relevant couplings only. Nevertheless, since the coupling $\lambda$ is only marginally irrelevant, the SU(2) Higgs model can be considered as an effective field theory with three non-zero renormalised couplings at finite values of the cut-off (the inverse lattice spacing $a$ ). In this article, we describe a method for studying the scaling properties of this effective field theory.

In practice, the LCPs in the $\mathrm{SU}(2)$ Higgs model are constructed by changing $\beta$ (which corresponds to a change in the lattice spacing) while renormalising the parameters $\kappa$ and $\lambda$ in order to keep two independent physical quantities $F_{1}$ and $F_{2}$ constant:

$$
F_{i}(\beta, \kappa, \lambda)=\text { constant }, \quad i=1,2 \text {. }
$$

Along a LCP, the value of a general physical quantity $F$ (different than $F_{1}$ and $F_{2}$ ) shows a dependency on the lattice spacing like [4,5]

$$
\left.F(a)\right|_{F_{1}, F_{2}}=\bar{F}+\mathrm{O}\left(\left(a / r_{0}\right)^{2}\right)
$$

where $\bar{F}$ is independent of $a$ and $r_{0}$ is the physical scale, like the Sommer scale [6]. The $\mathrm{O}\left(\left(a / r_{0}\right)^{2}\right)$ terms arise from irrelevant operators and are called lattice artifacts. The absence of $\mathrm{O}\left(a / r_{0}\right)$ corrections on the right-hand side of eq. (2) for a scalar theory is well accepted. Because the renormalised coupling $\lambda_{\mathrm{R}}$ vanishes only logarithmically along a LCP [7], i.e. like $1 / \ln \left(r_{0} / a\right)$, it is possible to find a range of values of the lattice spacing where the corrections on the right-hand side of eq. (2) are negligible but $\lambda_{\mathrm{R}}$ is still sizeable. In this range, which we refer to as the scaling region, for a given lattice spacing the bare coupling $\lambda$ can be tuned such that $\lambda_{\mathrm{R}}$ assumes a particular value: the effective field theory described by the $\mathrm{SU}(2)$ Higgs model with finite cut-off has three independent couplings.

The motivation behind the work presented in this article is the following. In a gauge theory with matter fields in the fundamental representation of the gauge group, 
the potential between a static quark and a static anti-quark 1 is expected to flatten at large separation of the static sources. This phenomenon is called string breaking [8]. Whereas string breaking has not been observed in QCD simulations yet [9, 10], it has been established by numerical simulations of the four-dimensional [11] and threedimensional 12] $\mathrm{SU}(2)$ Higgs model, where a new method for determining the static potential has been used. The phase diagram of the SU(2) Higgs model [3] is divided into two "phases": the Higgs "phase" at large values of $\kappa$ and the confinement "phase" at smaller $\kappa$. In the confinement "phase", the model has confinement properties like QCD [1,13] and is therefore suitable for testing the method of determining the static potential. In order to investigate the size of scaling violations in the measurements of the static potential a way to define LCPs in the confinement "phase" of the SU(2) Higgs model has to be found. We anticipate that our results for the static potential in the four-dimensional SU(2) Higgs model [14,15] show compatibility with scaling within tiny errors already at moderate $\beta$ values.

In three space-time dimensions, the LCPs can be exactly determined within perturbation theory [16] due to super-renormalisability of the theory. In the four-dimensional model, which we consider in this article, the variations of $\kappa$ and $\lambda$ along the LCP have to be determined non-perturbatively. We construct two physical quantities, $F_{1}$ and $F_{2}$, which have a sufficiently independent sensitivity on $\kappa$ and $\lambda$ in order to be taken for the definition of a LCP. The quantity $F_{1}$ is constructed from the static potential and is sensitive to the mass of the Higgs field which determines the string breaking distance $r_{\mathrm{b}}$. The quantity $F_{2}$ is a generalised Binder cumulant constructed from the connected $p$-point functions of a zero-momentum gauge-invariant Higgs field: it has proven to be sensitive enough to the Higgs quartic self-coupling that we can use it to construct a LCP. We present numerical results for the renormalisation (or matching) of the bare parameters along a LCP between $\beta=2.2$ and $\beta=2.4$.

\section{Definitions}

A sensible choice for the physical quantities $F_{1}$ and $F_{2}$, which allows us to match the parameters $\kappa$ and $\lambda$ along a LCP, would be to find an $F_{1}$ which is strongly dependent on $\kappa$ and an $F_{2}$ which is strongly dependent on $\lambda$. In this sense, a good quantity $F_{1}$ can be defined as

$$
F_{1}=r_{0}\left[2 \mu-V_{0}\left(r_{0}\right)\right],
$$

where $V_{0}(r)$ is the static potential and $\mu$ is the mass of a static-light meson, the lowest bound state of a static quark and the light dynamical Higgs field. The difference $2 \mu-$ $V_{0}(r)$ is a renormalised quantity free of self-energy contributions of the static sources,

\footnotetext{
${ }^{1}$ A static quark (anti-quark) is an external source in the (complex conjugate of the) fundamental representation of the gauge group.

2 At small values of $\beta$ there is an analytic connection between the two "phases": therefore, we put "phase" in quotation marks.
} 
which diverge in the continuum like $a^{-1}$. Because the static potential flattens reaching its asymptotic value $\lim _{r \rightarrow \infty} V_{0}(r)=2 \mu$, the quantity $F_{1}$ eq. (3) is strictly related to the string breaking distance $r_{\mathrm{b}}$, around which the static potential starts flattening out, and is mainly sensitive to the value of $\mu$ and hence to $\kappa$, which determines the mass of the Higgs field.

A remark should be added concerning finite size effects. Because physics shows a dependence on the physical size of the system, the latter is part of the definition of a physical quantity $F$. Along a LCP, the physical size of the lattice $L / r_{0}$ has either to be kept constant or at least large enough to avoid finite size effects. As concerns the static potentials and the static-light meson spectrum, the relevant quantities to compare are the lattice size $L$ and the mass gap in the gauge invariant sector of the theory, the Higgs mass $m_{\mathrm{H}}$. If $L m_{\mathrm{H}} \gg 1$ finite size effects are expected to be negligible. This condition is fulfilled in our numerical computations [14].

Now, we are looking for a physical quantity $F_{2}$ which is sensitive to a variation of the parameter $\lambda$, once the parameter $\kappa$ is matched by using $F_{1}$ eq. (3). There exist perturbative equations [7] describing the change of $\lambda$ and $g$ along a LCP in the weak coupling limit. But the use of these equations is not justified in our case because the bare couplings are not small enough. The change of $\lambda$ along a LCP has to be computed in a non-perturbative way. Exploratory results [1] indicated that the physics of the SU(2) Higgs model in the confinement "phase" is weakly dependent on $\lambda$ once a physical quantity such as $F_{1}$ eq. (3) is kept fixed. Our studies of correlation functions from which the Higgs and W-boson mass can be extracted confirm these observations [14].

Here, we consider a zero-momentum gauge-invariant Higgs field

$$
s=\frac{1}{\Omega} \sum_{x} S(x)=\frac{1}{\Omega} \sum_{x} \Phi^{\dagger}(x) \Phi(x),
$$

where $\Phi(x)$ is the complex $\mathrm{SU}(2)$ doublet Higgs field and $\Omega$ is the number of lattice points. The field $S(x)$ is a composite field. The renormalisation of composite fields is a complicated issue and we refer to textbooks, e.g [17,18], for a detailed discussion. The field $S(x)$ gets renormalised like $S_{\mathrm{R}}(x)=Z_{1}+Z_{S} S(x)$. If one considers the connected $p$-point functions $G_{c, \mathrm{R}}\left(x_{1}, \ldots, x_{p}\right)=\left\langle S_{\mathrm{R}}\left(x_{1}\right) \cdots S_{\mathrm{R}}\left(x_{p}\right)\right\rangle_{c}$, the additive renormalisation $Z_{1}$ cancels and the multiplicative renormalisation $Z_{S}$ is chosen such that the functions $G_{c, \mathrm{R}}\left(x_{1}, \ldots, x_{p}\right)$ have a well-defined continuum limit, provided the arguments $x_{1}, \ldots, x_{p}$ are kept at non-zero distance from one another [19].

Additional care is required if some of the arguments $x_{1}, \ldots, x_{p}$ in $G_{c, \mathrm{R}}\left(x_{1}, \ldots, x_{p}\right)$ coincide, because in this case divergences can arise in taking the continuum limit. This situation can be analysed in the continuum with the help of the operator-product expansion of Wilson [20], according to which

$$
\left\langle S_{\mathrm{R}}\left(x_{1}\right) \cdots S_{\mathrm{R}}\left(x_{k}\right)\right\rangle_{c} \underset{x_{i}-x_{j} \rightarrow \epsilon}{\sim} \frac{1}{|\epsilon|^{2 k}} \quad(\epsilon \rightarrow 0),
$$

in the limit of all coincident arguments. The power of the divergence on the right-hand side of eq. (5) corresponds to the naive dimensional counting. When we take the fields 


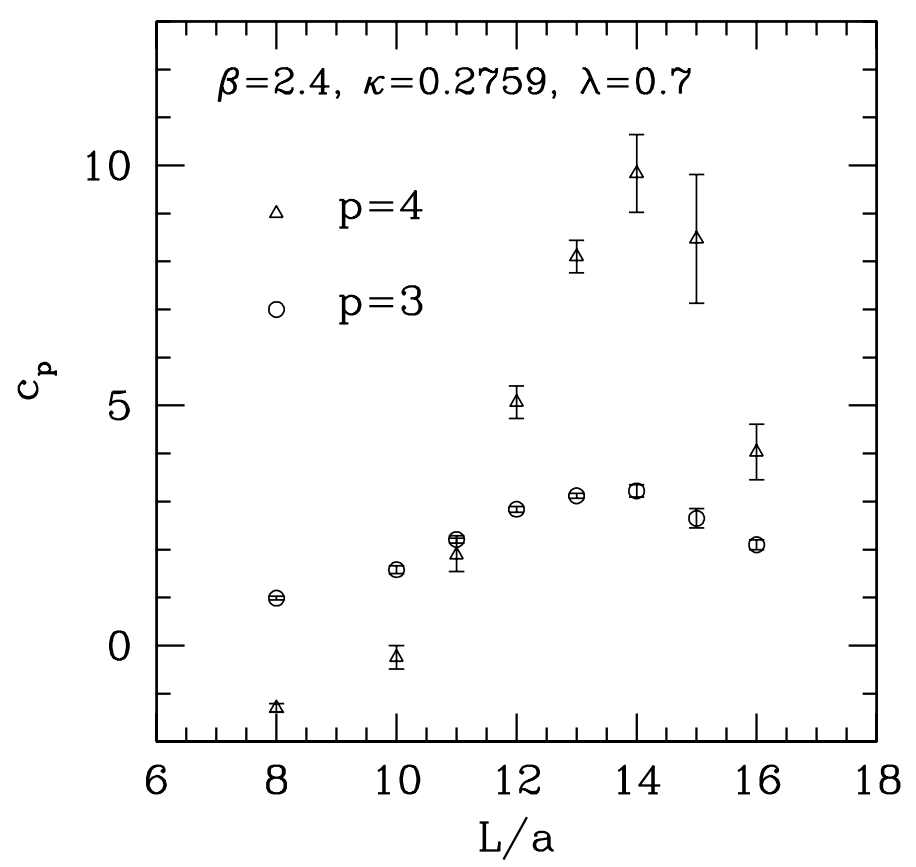

Figure 1: The cumulants $c_{3}$ and $c_{4}$ defined in eq. (8) as functions of the lattice size at $\beta=2.4$.

$S_{\mathrm{R}}(x)$ in eq. (5) at zero momentum we get the expression

$$
\begin{aligned}
\left\langle s_{\mathrm{R}}^{p}\right\rangle_{c} & =\frac{1}{V^{p}} \int \mathrm{d}^{4} x_{1} \cdots \mathrm{d}^{4} x_{p}\left\langle S_{\mathrm{R}}\left(x_{1}\right) \cdots S_{\mathrm{R}}\left(x_{p}\right)\right\rangle_{c} \\
& =\frac{1}{V^{p-1}} \int \mathrm{d}^{4} y_{1} \cdots \mathrm{d}^{4} y_{p-1}\left\langle S_{\mathrm{R}}\left(y_{1}\right) \cdots S_{\mathrm{R}}\left(y_{p-1}\right) S_{\mathrm{R}}(0)\right\rangle_{c},
\end{aligned}
$$

where $V$ is the physical volume of the torus and in the second line we used the translation invariance property of $G_{c, \mathrm{R}}\left(x_{1}, \ldots, x_{p}\right)$. From eq. (5) and naive dimensional counting, we infer that in the limit when $y_{i} \rightarrow 0(i=1, \ldots, p-1)$ in eq. (6) the integral is finite except in the case $p=2$ where it is naively logarithmic divergent. This problem can be cured by defining a modified connected 2-point function

$$
\left\langle\bar{s}_{\mathrm{R}}^{(2)}\right\rangle_{c}=\frac{1}{V} \int \mathrm{d}^{4} y\left\langle S_{\mathrm{R}}(y) S_{\mathrm{R}}(0)\right\rangle_{c} \sin \left(\frac{y_{0}}{T} \pi\right)
$$

where $T$ is the time extension of the torus: we use $T \equiv L \equiv V^{1 / 4}$. The sine function gives a contribution proportional to $y_{0}$ when $y \rightarrow 0$ and therefore it makes the integral finite. In order to be sure that all divergences associated with coincident arguments in eq. (16) are regularised for $p>2$, it remains to consider the case of two coincident arguments, e.g. $y_{1} \rightarrow y_{2}$. In contrast with the case $p=2$, there are still two integrations 


\begin{tabular}{|c|c|c||c|c|c|}
\hline$\lambda$ & $\kappa$ & $\Delta \kappa$ & $\lambda$ & $\kappa$ & $\Delta \kappa$ \\
\hline \hline 0.4 & 0.2615 & 0.0001 & 0.757 & 0.2973 & 0.0001 \\
\hline 0.5 & 0.2737 & 0.0001 & 0.96 & 0.3108 & 0.0001 \\
\hline 0.55 & 0.2792 & 0.0002 & 1.0 & 0.3131 & 0.0001 \\
\hline 0.7 & 0.2929 & 0.0001 & 1.5 & 0.3351 & 0.0002 \\
\hline
\end{tabular}

Table 1: Values of $\kappa$ for different $\lambda$ 's determined at $\beta=2.2$ by using the matching condition eq. (11). The values of $\Delta \kappa$ are the uncertainties associated with the matching.

over $y_{1}$ and $y_{2}$ and the integral is finite. We can now define generalised Binder cumulants [21] $c_{p}$ as

$$
c_{p}=\frac{\left\langle s^{p}\right\rangle_{c}}{\left[\left\langle\bar{s}^{(2)}\right\rangle_{c}\right]^{p / 2}} \quad(p=3,4, \ldots) .
$$

From the above discussion, it follows that the couplings $c_{p}$ are well-defined and renormalised. Notice that the bare fields can be used in the definition of eq. (8) because the multiplicative renormalisation factors $Z_{S}$ cancel.

The cumulants $c_{p}$ can be defined on the lattice using the same expression eq. (8): the field $s$ is given in eq. (4) and

$$
\left\langle\bar{s}^{(2)}\right\rangle_{c}=\frac{1}{\Omega^{2}} \sum_{x, y}\langle S(x) S(y)\rangle_{c} \sin \left(\frac{x_{0}-y_{0}}{T} \pi\right)
$$

is the lattice version of eq. (7). In the next section, we present Monte Carlo results of the computation of the cumulants $c_{p}$ on the lattice which show a linear sensitivity to a variation of the parameter $\lambda$, once the physical quantity $F_{1}$ eq. (3) is kept fixed. We define the second physical quantity $F_{2}$ characterising the LCP to be

$$
F_{2}=\left.c_{3}\right|_{L / r_{0}},
$$

where the physical size $L / r_{0}$ of the lattice on which $c_{3}$ is computed has to be kept constant along the LCP. This is required because the cumulants $c_{p}$ are finite size couplings, as we are going to see in the next section.

Our final result is that physics in the confinement "phase" of the $\mathrm{SU}(2)$ Higgs Model can be reproduced at different values of the lattice spacing by changing the bare parameters $\kappa$ and $\lambda$ such that the physical quantities $F_{1}$ eq. (3) and $F_{2}$ eq. (10) are kept constant. In the next section, we construct two points on a LCP at $\beta$ values 2.2 and 2.4 by computing $F_{1}$ and $F_{2}$ in Monte Carlo simulations.

\section{Numerical results}

At $\beta=2.4$, we take the parameter set $\kappa=0.2759, \lambda=0.7$ [14 and we determine the corresponding values $F_{1}^{*}$ and $F_{2}^{*}$ (referred as matching conditions) of the physical quan- 


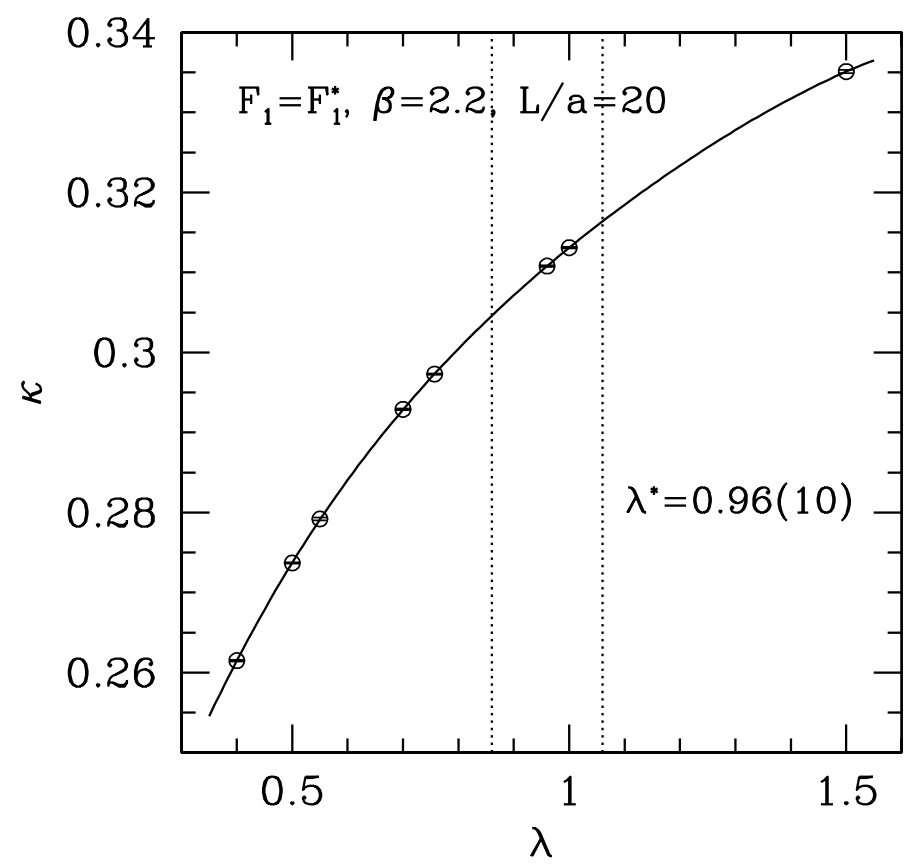

Figure 2: The curve $\kappa(\lambda)$ at $\beta=2.2$ from the matching condition eq. (11). The solid line represents the polynomial fit eq. (15). The vertical dotted band represents the matched value of $\lambda$ (with error) along the LCP eq. (17).

tities $F_{1}$ eq. (3) and $F_{2}$ eq. (10) that define the LCP. From the quantity $F_{1}$, computed on a $32^{4}$ lattice, we obtain the first matching condition

$$
F_{1}=F_{1}^{*} \equiv 1.26
$$

with a statistical error $\Delta F_{1}^{*}=0.02$.

The cumulants $c_{p}$ defined in eq. (8) are very small for large values of $p$ : in the Monte Carlo simulations, we were able to obtain a significant signal up to $p=5$. In a massive theory, from the translation invariance property of connected $p$-point functions (see eq. (6) ) the cumulants are expected to scale for large volumina $V$ of the torus like

$$
c_{p} \propto\left(m_{\mathrm{H}}^{4} V\right)^{1-p / 2},
$$

where $m_{\mathrm{H}}$ is the Higgs mass. We computed $c_{3}$ and $c_{4}$ at $\beta=2.4$ for different lattice sizes: the results are shown in Fig. 1. We observe strong variations and therefore, a precise matching of the physical lattice size $L / r_{0}$ along the LCP is needed in order to use $F_{2}=c_{3}$ for defining the LCP. We choose to compute $c_{3}$ on a $7^{4}$ lattice at $\beta=2.2$. The physical lattice size is small enough to obtain a good signal and the matched lattice size 


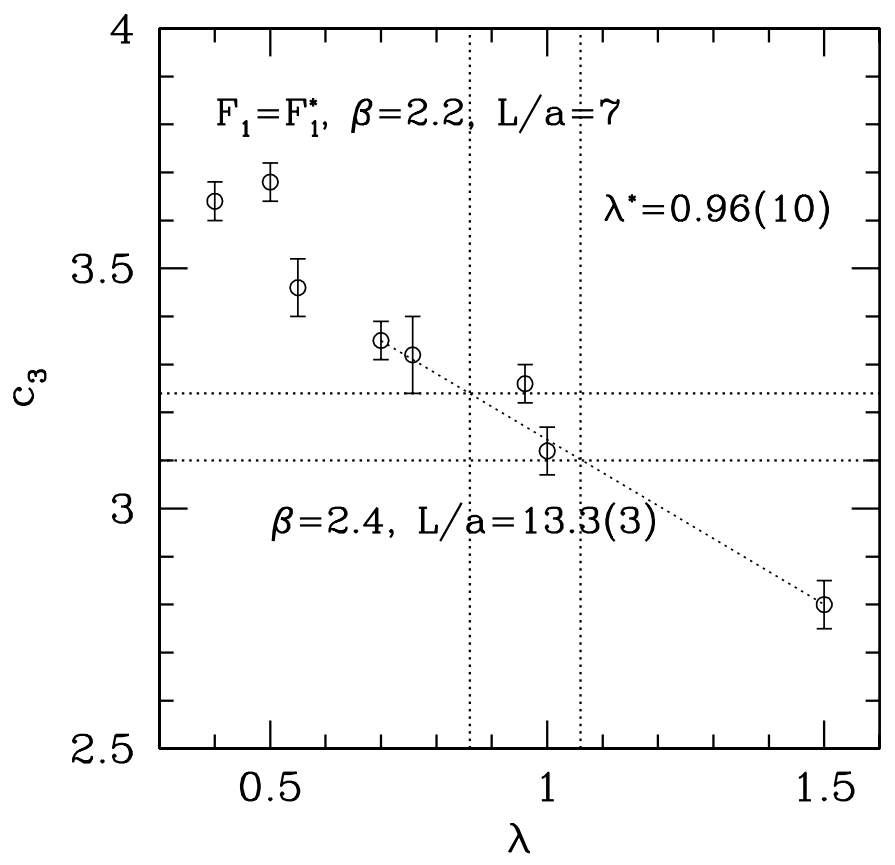

Figure 3: The $\lambda$-dependence of the cumulant $c_{3}$ at $\beta=2.2$. The values for the parameter $\kappa$ are the same as in Table 1. The horizontal dotted band represents the second matching condition eq. (13). From the slope of $c_{3}(\lambda)$, we determine the matched value $\lambda^{*}$.

at $\beta=2.4$ is $L / a=13.3(3)$ 自 in a region where $c_{3}$ has no strong variations (see Fig. 1). Interpolating the values of $c_{3}$ at $\beta=2.4$ shown in Fig. 1 to the matched lattice size gives the second matching condition

$$
F_{2}=F_{2}^{*} \equiv 3.17
$$

with an error $\Delta F_{2}^{*}=0.07$.

Now, we are ready to match the parameters $\kappa$ and $\lambda$ at $\beta=2.2$. We start with matching $\kappa$ for different values of $\lambda$ by computing $F_{1}$ on a $20^{4}$ lattice and requiring eq. (11) to be fulfilled. The resulting $(\lambda, \kappa)$ pairs are listed in Table 1 and plotted in Fig. 2. We fit these points with a polynomial

$$
\kappa(\lambda)=\sum_{k=0}^{M} a_{k}(\lambda-1)^{k} .
$$

A good description of our data is obtained for $M=4$ :

$$
\begin{aligned}
\kappa(\lambda)= & 0.3131+0.0564(\lambda-1)-0.0286(\lambda-1)^{2} \\
& +0.0198(\lambda-1)^{3}-0.0246(\lambda-1)^{4},
\end{aligned}
$$

\footnotetext{
${ }^{3}$ The error comes from the determination of $r_{0} / a$ at the two $\beta$ values.
} 


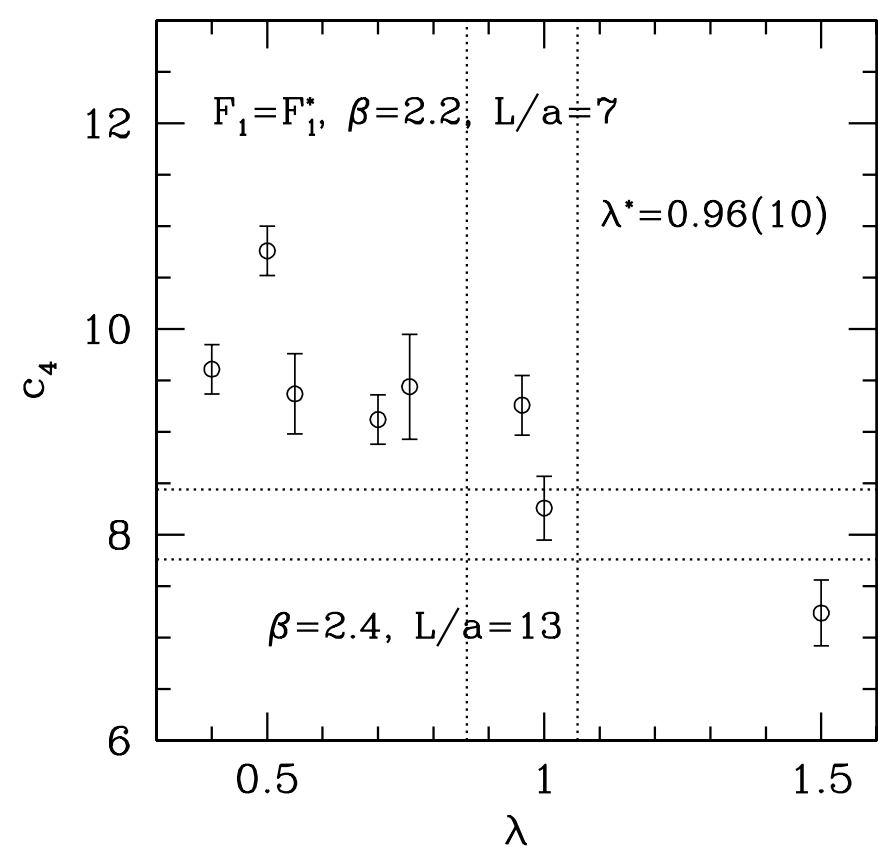

Figure 4: The $\lambda$-dependence of the cumulant $c_{4}$ at $\beta=2.2$. The values for the parameter $\kappa$ are the same as in Table 1. The horizontal dotted line is the value of $c_{4}$ at $\beta=2.4$ for only approximately matched physical lattice size. The vertical dotted line is the matched value $\lambda^{*}$ along the LCP eq. (17).

and a comparison of this expression with the data points is shown in Fig. 2. The deviation between the fitted curve eq. (15) and the data points is smaller than the statistical errors of the data. When $\kappa$ is evaluated using eq. (15), an uncertainty of $1 \cdot 10^{-4}$ should be assigned in the range $0.4 \leq \lambda \leq 1.06$ growing up to $4 \cdot 10^{-4}$ in the range $1.06<\lambda \leq 1.5$. As a by-product of the simulations for the matching of $\kappa$ we obtain for the scale $r_{0}$ the typical value $r_{0} / a \approx 2.8$ when $F_{1}$ takes values near $F_{1}^{*}$. This has to be compared with the value $r_{0} / a=5.29(6)$ obtained at $\beta=2.4$ : the resulting change in the lattice spacing is

$$
\left.\frac{a(\beta=2.2)}{a(\beta=2.4)}\right|_{F_{1}=F_{1}^{*}}=1.90(4),
$$

where at $\beta=2.2$ we take $\lambda=1.0$.

The matching of $\lambda$ at $\beta=2.2$ is performed by computing $F_{2}=c_{3}$ on a $7^{4}$ lattice taking the parameter points $(\lambda, \kappa)$ of Table 1 . The results are shown in Fig. 3. The matched value $\lambda^{*}$ is determined by requiring the matching condition eq. (13) (represented in Fig. 3 by the horizontal dotted band) to be fulfilled: we obtain $\lambda^{*}=0.96(10)$, 
where the error is determined from the approximate slope of $c_{3}(\lambda)$. Summarising our numerical results, the points

$$
\begin{array}{cc}
\beta=2.2, \quad \kappa^{*}=\kappa\left(\lambda^{*}\right), & \lambda^{*}=0.96(10) \\
& \text { and } \\
\beta=2.4, \quad \kappa=0.2759, \quad \lambda=0.7
\end{array}
$$

lie on the LCP defined by the matching conditions eq. (11) and eq. (13). The value of $\kappa$ at $\beta=2.2$ is determined using the fit eq. (15).

In Fig. 1, we show the behavior of the cumulant $c_{4}$ on the LCP eq. (17). The horizontal dotted band is the approximate value of $c_{4}$ at $\beta=2.4$ : the physical lattice size is only approximately matched. The values of $c_{4}$ at $\beta=2.2$ when $\lambda$ is in the matched interval around $\lambda^{*}$ are compatible with scaling.

\section{Conclusions}

We have described a non-perturbative method for matching the parameters $\kappa$ and $\lambda$ along a LCP in the confinement "phase" of the SU(2) Higgs model. We consider the model at finite values of the cut-off, where it describes an effective field theory with a non-zero renormalised Higgs quartic self-coupling. The lattice spacing is varied by changing $\beta$ while two physical quantities $F_{1}$ and $F_{2}$ are kept constant. A requirement for $F_{1}$ and $F_{2}$ is that they have a sufficiently independent sensitivity to $\kappa$ and $\lambda$. The first quantity $F_{1}$ is derived from the static potential and is mainly sensitive to $\kappa$. More problematic is the definition of the second quantity $F_{2}$ because the physics in the confinement "phase" is weakly dependent on $\lambda$. We construct couplings, which are generalised Binder cumulants, from the connected $p$-point functions of a zero-momentum gauge-invariant Higgs field. In particular, we define a modified 2-point function in order that no divergencies arise in taking the continuum limit. In the range of $\lambda$ that we investigated numerically, these couplings show a linear dependence on $\lambda$ that allows for the matching. We determine by Monte Carlo simulations a LCP at $\beta$ values 2.2 and 2.4. Based on these results, it is possible to study the scaling properties of physical quantities such as the static potential [15].

Acknowledgement. I thank B. Bunk, R. Sommer, A. Hasenfratz and T. DeGrand for helpful discussions, the Konrad-Zuse-Zentrum für Informationstechnik Berlin (ZIB) for granting CPU-resources to this project and DESY Zeuthen, where most of this work was done.

\section{References}

[1] I. Montvay, Nucl. Phys. B269 (1986) 170.

[2] W. Langguth, I. Montvay and P. Weisz, Nucl. Phys. B277 (1986) 11. 
[3] For further discussion, see for example I. Montvay and G. Münster, Quantum Fields on a Lattice, Cambridge University Press (1994).

[4] K. Symanzik, Nucl. Phys. B226 (1983) 187.

[5] K. Symanzik, Nucl. Phys. B226 (1983) 205.

[6] R. Sommer, Nucl. Phys. B411 (1994) 839, hep-lat/9310022.

[7] I. Montvay, Nucl. Phys. B293 (1987) 479.

[8] For a review see K. Schilling, to appear in the proceedings of the 17th International Symposium on Lattice Field Theory (Pisa 1999), Nucl. Phys. B Proc. Suppl. , heplat/9909152.

[9] CP-PACS, R. Burkhalter et al., Nucl. Phys. Proc. Suppl. 73 (1999) 3, heplat/9810043.

[10] CP-PACS-Collaboration, S. Aoki et al., (1999), hep-lat/9902018.

[11] ALPHA-Collaboration, F. Knechtli and R. Sommer, Phys. Lett. B440 (1998) 345, erratum: Phys. Lett. B454 (1999) 399, hep-lat/9807022.

[12] O. Philipsen and H. Wittig, Phys. Rev. Lett. 81 (1998) 4056, hep-lat/9807020.

[13] H.G. Evertz et al., Phys. Lett. 175B (1986) 335.

[14] F. Knechtli, PhD thesis at Humboldt-University Berlin (1999), hep-lat/9910044.

[15] F. Knechtli and R. Sommer, in preparation. See also F. Knechtli, to appear in the proceedings of the 17th International Symposium on Lattice Field Theory (Pisa 1999), Nucl. Phys. B Proc. Suppl., hep-lat/9909164 .

[16] M. Laine, Nucl. Phys. B451 (1995) 484, hep-lat/9504001.

[17] J. Collins, Renormalization, Cambridge University Press (1995).

[18] T. Muta, Foundations of Quantum Chromodynamics, World Scientific (1987).

[19] For a pedagogic survey of this point, see M. Lüscher, Advanced lattice QCD, talk given at Les Houches Summer School in Theoretical Physics (1997), heplat/9802029, plus references therein.

[20] K.G. Wilson, Phys. Rev. 179 (1969) 1499.

[21] K. Binder, Z. Phys. B43 (1981) 119. 